TRANSACTIONS OF THE

AMERICAN MATHEMATICAL SOCIETY

Volume 349, Number 4, April 1997, Pages 1421-1436

S 0002-9947(97)01478-5

\title{
CONVEX INTEGRAL FUNCTIONALS
}

\author{
NIKOLAOS S. PAPAGEORGIOU
}

\begin{abstract}
We study nonlinear integral functionals determined by normal convex integrands. First we obtain expressions for their convex conjugate, their $\varepsilon$-subdifferential $(\varepsilon \geq 0)$ and their $\varepsilon$-directional derivative. Then we derive a necessary and sufficient condition for the existence of an approximate solution for the continuous infimal convolution. We also obtain general conditions which guarantee the interchangeability of the conditional expectation and subdifferential operators. Finally we examine the conditional expectation of random sets.
\end{abstract}

\section{INTRODUCTION}

In many areas of applied mathematics, such as control theory, mathematical economics and stochastic nonlinear programming, we have to deal with integral functionals of the form $I_{\varphi}(x)=\int_{\Omega} \varphi(\omega, x(\omega)) d \mu(\omega)$, where $(\Omega, \Sigma, \mu)$ is a measure space, $\varphi: \Omega \times X \rightarrow \overline{\mathbb{R}}=\mathbb{R} \cup\{+\infty\}$ is a measurable integrand, $X$ a Banach space and $x(\cdot)$ belongs to a space of measurable $X$-valued functions on $\Omega$.

Classically only finite Carathéodory-type integrands $\varphi(\omega, x)$ were considered (i.e. $\omega \rightarrow \varphi(\omega, x)$ measurable and $x \rightarrow \varphi(\omega, x)$ continuous $)$. However in many important applications $\varphi(\omega, \cdot)$ is discontinuous and it is important to allow $\varphi(\cdot, \cdot)$ to take infinite values, since this way we can represent more efficiently several types of constraints. In this situation convexity provides the necessary framework to develop a complete theory of such integral functionals. This line of research was initiated by Rockafellar [21, 22, 23], who introduced the notion of "normal integrand", which proved to be a very fruitful concept for the study of $I_{\varphi}(\cdot)$. Subsequent important contributions were made by Ioffe-Tihomirov [12], Rockafellar [24], Ioffe-Levin [11], Bismut [4], Levin [18], Hiriart-Urruty [9], Castaing-Valadier [6] and Komuro [15]. A well written, comprehensive introduction to the subject when $X=\mathbb{R}^{n}$ can be found in Rockafellar [25].

The main contribution of this work is to extend and improve certain formulas concerning basic operations from convex analysis to normal integrands defined on $\Omega \times X$ with $X$ being a separable Banach space. Previously those results were known for integrands defined on $\Omega \times \mathbb{R}^{n}$. Moreover, in certain cases (see for example, the results on commutation of conditional expectation and subdifferentiation in section 5) our approach is different from the one existing in the literature and is based on some intermediate results which we believe are of independent interest. In the next

Received by the editors January 13, 1994 and, in revised form, January 23, 1995.

1991 Mathematics Subject Classification. Primary 47H30, 49N15.

Key words and phrases. Normal convex integrand, $\varepsilon$-subdifferential, multifunction, support function, singular functional, conditional expectation, Souslin space subdifferential.

(C)1997 American Mathematical Society 
paragraph we specify in more detail the contributions of this work and its relation to the literature.

Our work here extends and complements some of the results in the abovementioned papers. In particular in section 3 , we look at the $\varepsilon$-subdifferential of the functional $x \rightarrow \Phi(x)=\int_{\Omega} \varphi(\omega, x) d \mu(\omega)$ and derive a formula for it in terms of the $\varepsilon$-subdifferentials of the functionals $\varphi(\omega, \cdot), \omega \in \Omega$. Our result extends the corresponding ones obtained by Hiriart-Urruty [9] and Komuro [15] for integrands $\varphi(\omega, x)$ defined on $\Omega \times \mathbb{R}^{n}$. Also when $\varepsilon=0$, we recover the formula for the subdifferential $\partial \Phi(x)$ originally due to Rockafellar [24]. Our proof is based on a result concerning the convex conjugate of $\Phi(\cdot)$ which extends an analogous result proved by Valadier [30] for $X=\mathbb{R}^{n}$ and Rockafellar [24] for $X=$ separable reflexive Banach space. In section 4 , we examine the operation of continuous infimal convolution and using the $\varepsilon$-subdifferential of the integrand $\varphi(\omega, x)$, we obtain a necessary and sufficient condition for the existence of an $\varepsilon$-optimal solution. The operation of continuous infimal convolution arises naturally in mathematical economics in the study of transferable utilities (see Aumann-Shapley [3]). We also use the formula for the $\varepsilon$-subdifferential of $\Phi(x)=\int_{\Omega} \varphi(\omega, x) d \mu(\omega)$ proved in section 3, in order to describe the level sets of the continuous infimal convolution. In the past, this operation and its applications in stochastic optimization were studied primarily by Hiriart-Urruty [9] in the context of integrands defined on $\Omega \times \mathbb{R}^{n}$. In section 5 , we deal with the interchange of the operations of conditional expectation and subdifferentiation. We give a condition which guarantees that the two operations commute when applied to convex integral functionals. Our result extends a similar one proved by Rockafellar-Wets [27], using a different approach for functionals $\varphi(\omega, x)$ defined on $\Omega \times \mathbb{R}^{n}$. Such a result can be useful in problems of stochastic optimization, like multistage stochastic programming problems. Finally in section 6 , we consider the conditional expectation of a random set and examine its support and indicator functions. Our results can be used in the study of the convergence properties of set-valued stochastic processes.

\section{Preliminaries}

Let $(\Omega, \Sigma)$ be a measurable space and $X$ a separable Banach space. By $P_{f(c)}(X)$ we will denote the nonempty, closed (and convex) subsets of $X$. A multifunction (set-valued function) $F: \Omega \rightarrow P_{f}(X)$ is said to be measurable, if for every $z \in X$ the $\mathbb{R}_{+}$-valued function $\omega \rightarrow d(z, F(\omega))=\inf \{\|z-x\|: x \in F(\omega)\}$ is measurable. This implies that $\operatorname{Gr} F=\{(\omega, x) \in \Omega \times X: x \in F(\omega)\} \in \Sigma \times B(X)$, with $B(X)$ being the Borel $\sigma$-field of $X$ (graph measurability). The converse is true if $\Sigma$ is complete; i.e. $\Sigma=\widehat{\Sigma}$ with $\widehat{\Sigma}$ being the universal $\sigma$-field generated by $\Sigma$. Recall that if $\mu(\cdot)$ is a $\sigma$-finite measure on $\Sigma$ and $\Sigma$ is $\mu$-complete, then $\Sigma=\widehat{\Sigma}$ (see Wagner [31]).

Now let $(\Omega, \Sigma, \mu)$ be a $\sigma$-finite measure space and $F: \Omega \rightarrow 2^{X} \backslash\{\varnothing\}$. We define

$$
S_{F}^{1}=\left\{f \in L^{1}(\Omega, X): f(\omega) \in F(\omega) \mu \text {-a.e. }\right\} ;
$$

i.e. $S_{F}^{1}$ contains all integrable selectors of $F(\cdot)$. The set $S_{F}^{1}$ may be empty. An easy application of Aumann's selection theorem (see Wagner [31], Theorem 5.10) reveals that, for a graph measurable $F(\cdot), S_{F}^{1} \neq \varnothing$ if and only if $\omega \rightarrow \inf [\|x\|: x \in F(\omega)] \in$ $L^{1}(\Omega)$. If $F(\cdot)$ is $P_{f(c)}(X)$-valued, then $S_{F}^{1}$ is closed (and convex) in $L^{1}(\Omega, X)$ and it is bounded if and only if $\sup [\|x\|: x \in F(\omega)] \leq \theta(\omega) \mu$-a.e. with $\theta(\cdot) \in L^{1}(\Omega)$ 
(i.e. $F(\cdot)$ is integrably bounded). Using this set, we can define a set-valued integral for $F(\cdot)$ by setting

$$
\int_{\Omega} F(\omega) d \mu(\omega)=\left\{\int_{\Omega} f(\omega) d \mu(\omega): f \in S_{F}^{1}\right\} .
$$

This integral was first introduced by Aumann [2] for $X=\mathbb{R}^{n}$ and is a natural generalization of the integral of single-valued functions and of the Minkowski sum of sets.

Now assume that $(\Omega, \Sigma, \mu)$ is a probability space and $\Sigma_{0} \subseteq \Sigma$ a sub- $\sigma$-field. From Hiai-Umegaki [8] we know that given a measurable multifunction $F: \Omega \rightarrow P_{f}(X)$ with $S_{F}^{1} \neq \varnothing$, there exists a unique (up to sets of $\mu$-measure zero), $\Sigma_{0}$-measurable multifunction $E^{\Sigma_{0}} F: \Omega \rightarrow P_{f}(X)$ (called the conditional expectation of $F(\cdot)$ with respect to $\left.\Sigma_{0}\right)$ such that $S_{E^{\Sigma_{0} F}}^{1}\left(\Sigma_{0}\right)=\left\{g \in L^{1}\left(\Sigma_{0}, X\right): g(\omega) \in E^{\Sigma_{0}} F(\omega) \mu\right.$-a.e. $\}=$ $\operatorname{cl}\left\{E^{\Sigma_{0}} f: f \in S_{F}^{1}\right\}$, the closure taken in $L^{1}(\Omega, X)$.

Let $\varphi: X \rightarrow \overline{\mathbb{R}}=\mathbb{R} \cup\{+\infty\}$ be a function. The effective domain and epigraph of $\varphi(\cdot)$ are defined respectively by

$$
\operatorname{dom} \varphi=\{x \in X: \varphi(x)<+\infty\} \quad \text { and } \quad \operatorname{epi} \varphi=\{(x, \lambda) \in X \times \mathbb{R}: \varphi(x) \leq \lambda\} .
$$

We say that $\varphi(\cdot)$ is proper if and only if $\operatorname{dom} \varphi \neq \varnothing$. Also recall that $\varphi(\cdot)$ is lower semicontinuous (l.s.c.) if and only if epi $\varphi$ is closed in $X \times \mathbb{R}$. By $\Gamma_{0}(X)$ we will denote the set of all proper, l.s.c. and convex functions defined on $X$ with values in $\overline{\mathbb{R}}=\mathbb{R} \cup\{+\infty\}$. Given $\varphi: X \rightarrow \overline{\mathbb{R}}$ proper, the convex conjugate $\varphi^{*}: X^{*} \rightarrow \overline{\mathbb{R}}$ of $\varphi$ is defined by $\varphi^{*}\left(x^{*}\right)=\sup \left\{\left(x^{*}, x\right)-\varphi(x): x \in X\right\}$. Let $\varphi \in \Gamma_{0}(X)$. The subdifferential of $\varphi(\cdot)$ at $x$ is the set (possibly empty), $\partial \varphi(x)=\left\{x^{*} \in X^{*}:\left(x^{*}, y-\right.\right.$ $x) \leq \varphi(y)-\varphi(x)$ for all $y \in X\}=\left\{x^{*} \in X^{*}:\left(x^{*}, x\right)=\varphi(x)+\varphi^{*}\left(x^{*}\right)\right\}$. If $\varphi(\cdot)$ is Gateaux differentiable at $x$, then $\partial \varphi(x)=\{\nabla \varphi(x)\}$. In addition, if $\varphi(\cdot)$ is finite and continuous at $x$, then $\partial \varphi(x)$ is nonempty, convex and $w^{*}$-compact.

The $\varepsilon$-subdifferential $(\varepsilon \geq 0)$ of a proper function $\varphi: X \rightarrow \overline{\mathbb{R}}$ at $x$, is defined by

$$
\partial_{\varepsilon} \varphi(x)=\left\{x^{*} \in X^{*}:\left(x^{*}, y-x\right)-\varepsilon \leq \varphi(y)-\varphi(x) \text { for all } y \in X\right\} .
$$

We note that when $\varepsilon=0$ and $\varphi \in \Gamma_{0}(X)$, we recover the definition of the subdifferential. The $\varepsilon$-subdifferential enjoys some noteworthy properties that the exact subdifferential $(\varepsilon=0)$ lacks. Namely for $\varphi \in \Gamma_{0}(X)$ and $x \in \operatorname{dom} \varphi, \partial_{\varepsilon} \varphi(x) \in$ $P_{f_{c}}(X)$ (i.e. it is always nonempty) and $(x, \varepsilon) \rightarrow \partial_{\varepsilon} \varphi(x)$ is Hausdorff continuous on int dom $\varphi \times \overline{\mathbb{R}}_{+}$(cf. Hiriart-Urruty [10]). Roughly speaking, the reason for this striking difference is that while $\partial \varphi(\cdot)$ is a local notion, $\partial_{\varepsilon} \varphi(\cdot)$ is a global one.

Now let $(\Omega, \Sigma, \mu)$ be a complete, $\sigma$-finite measure space. An integrand $\varphi: \Omega \times$ $X \rightarrow \overline{\mathbb{R}}=\mathbb{R} \cup\{+\infty\}$ is said to be normal if (i) $(\omega, x) \rightarrow \varphi(\omega, x)$ is $\Sigma \times B(X)$ measurable, and (ii) for all $\omega \in \Omega, \varphi(\omega, \cdot) \in \Gamma_{0}(X)$. It is not difficult to see (cf. Rockafellar [23]), that this is equivalent to saying that (i) for every $\omega \in \Omega$, $\operatorname{epi} \varphi(\omega, \cdot) \in P_{f}(X)$ and (ii) ${ }^{\prime}$ the multifunction $\omega \rightarrow \operatorname{epi} \varphi(\omega, \cdot)$ is measurable. Note that a normal integrand is superpositionally measurable; i.e. if $x: \Omega \rightarrow X$ is measurable, then $\omega \rightarrow \varphi(\omega, x(\omega))$ is measurable. Also if $\varphi: \Omega \times X \rightarrow \mathbb{R}$ is a Carathéodory integrand (i.e. $\omega \rightarrow \varphi(\omega, x)$ is measurable and $x \rightarrow \varphi(\omega, x)$ is continuous), then $\varphi(\cdot, \cdot)$ is a normal integrand. We will say that $\varphi(\cdot, \cdot)$ is a normal convex integrand, if for all $\omega \in \Omega, \varphi(\omega, \cdot)$ is convex. 
Let $\varphi: \Omega \times \overline{\mathbb{R}}$ be a normal integrand. We define $I_{\varphi}: L^{p}(\Omega, X) \rightarrow \overline{\mathbb{R}}(1 \leq p \leq \infty)$ and $\Phi: X \rightarrow \overline{\mathbb{R}}$ by

$$
I_{\varphi}(x)= \begin{cases}\int_{\Omega} \varphi(\omega, x(\omega)) d \mu(\omega) & \text { if } \varphi(\cdot, x(\cdot))^{+} \in L^{1}(\Omega) \\ +\infty & \text { otherwise }\end{cases}
$$

and

$$
\Phi(x)= \begin{cases}\int_{\Omega} \varphi(\omega, x) d \mu(\omega) & \text { if } \varphi(\cdot, x)^{+} \in L^{1}(\Omega), \\ +\infty & \text { otherwise. }\end{cases}
$$

A functional $z \in L^{\infty}(\Omega, X)^{*}$ is said to be absolutely continuous with respect to $\mu(\cdot)$, if there exists a function $x^{*}(\cdot) \in L^{1}\left(\Omega, X_{w^{*}}^{*}\right)$ such that

$$
z(x)=\int_{\Omega}\left(x^{*}(\omega), x(\omega)\right) d \mu(\omega)
$$

for all $x \in L^{\infty}(\Omega, X)$. A functional $z \in L^{\infty}(\Omega, X)^{*}$ is said to be singular with respect to $\mu(\cdot)$ if there is a sequence $\left\{B_{m}\right\}_{m \geq 1} \subseteq \Sigma$ such that (i) $B_{m+1} \subseteq B_{m}$ for all $m \geq 1$, (ii) $\mu\left(B_{m}\right) \rightarrow 0$ as $m \rightarrow \infty$ and (iii) $z(x)=z\left(\chi_{B_{m}} x\right)$ for all $m \geq 1$ and all $x \in L^{\infty}(\Omega, X)$ (i.e. the sets $B_{m}$ support the functional $z$ ). We have the following decomposition theorem for $L^{\infty}(\Omega, X)^{*}$ due to Levin [18].

Theorem 1. Every functional $z \in L^{\infty}(\Omega, X)^{*}$ admits a unique decomposition $z=$ $z_{a}+z_{s}$ where $z_{a}$ is absolutely continuous with respect to $\mu(\cdot)$ and $z_{s}$ in singular with respect to $\mu(\cdot)$. Furthermore, $\|z\|=\left\|z_{a}\right\|+\left\|z_{s}\right\|$.

Given a normal integrand $\varphi: \Omega \times X \rightarrow \overline{\mathbb{R}}$, the infimal convolution of $\varphi(\omega, \cdot)$ with respect to $\mu(\cdot)$ is defined by

$$
\left(\oint_{\Omega} \varphi(\omega) d \mu(\omega)\right)(x)=\inf \left\{I_{\varphi}(v): v \in \mathcal{L}(x)\right\}
$$

where $x \in X$ and $\mathcal{L}(x)=\left\{v \in L^{1}(\Omega, X): x=\int_{\Omega} v(\omega) d \mu(\omega)\right\}$.

If $\varphi^{*}: \Omega \times X^{*} \rightarrow \overline{\mathbb{R}}$ is the convex conjugate of $\varphi(\omega, \cdot)$ (thus a normal integrand itself too), then the infimal convolution of $\varphi^{*}(\omega, \cdot)$ with respect to $\mu(\cdot)$, is defined by

$$
\left(\oint_{\Omega} \varphi^{*}(\omega) d \mu(\omega)\right)\left(x^{*}\right)=\inf \left\{I_{\varphi^{*}}\left(v^{*}\right): v^{*} \in \mathcal{L}\left(x^{*}\right)\right\}
$$

where $x^{*} \in X^{*}$ and $\mathcal{L}\left(x^{*}\right)=\left\{v^{*} \in L^{1}\left(\Omega, X_{w^{*}}^{*}\right): x^{*}=\int_{\Omega} v^{*}(\omega) d \mu(\omega)\right\}$. In the definition of $\mathcal{L}\left(x^{*}\right)$, the integral of $v^{*}(\cdot)$ is the Gelfand (or $w^{*}$-integral); cf. DiestelUhl [7], p. 53.

Let $V, W$ be Banach spaces, $A: V \rightarrow W$ a continuous linear operator and $g: W \rightarrow \mathbb{R} \cup\{ \pm \infty\}, f: V \rightarrow \mathbb{R} \cup\{ \pm \infty\}$ convex functions. Then we define (cf. Ioffe-Tihomirov [13], p. 169 and Rockafellar [22], p. 38)

$$
(g A)(v)=g(A v) \quad(\text { the inverse image of } g \text { under } A)
$$

and

$$
(A f)(w)=\inf \{f(v): v \in V, w=A v\} \quad \text { (the image of } f \text { under } A) .
$$

Finally, recall that a topological space $(Z, \tau)$ is a Polish space if and only if $\tau$ is metrizable for some metric $d$ such that $(Z, d)$ is complete and separable. A Hausdorff topological space $Z$ is said to be a Souslin space, if it is the continuous image of a Polish space. Clearly every Polish space is a Souslin space and also this 
is the case for a separable Banach space $X$ endowed with the weak topology. More generally, if $X$ is a separable Banach space, then $X^{*}$ equipped with the $\mathrm{w}^{*}$-topology is Souslin (cf. Bourbaki [5]). So a Souslin space need not be metrizable, but it is always separable. Also we mention that two comparable Souslin topologies generate the same Borel $\sigma$-field.

\section{The $\varepsilon$-SUBDIFFERENTIAL OF $\phi(\cdot)$}

In this section, $(\Omega, \Sigma, \mu)$ is a $\sigma$-finite, complete measure space and $X$ a separable Banach space. The completeness of $\mu(\cdot)$ is not actually necessary, but we have assumed it to simplify our exposition. The main result of this section is a formula for $\partial_{\varepsilon} \Phi(x)$.

We start by determining the convex conjugate of the integral functional $\Phi(x)=$ $\int_{\Omega} \varphi(\omega, x) d \mu(\omega)$. Our result extends Theorem 2.7 of Valadier [30] where $X=\mathbb{R}^{n}$ and Theorem 23(c) of Rockafellar [24], where $X=$ separable, reflexive Banach space.

Proposition 2. If $\varphi: \Omega \times X \rightarrow \overline{\mathbb{R}}=\mathbb{R} \cup\{+\infty\}$ is a normal convex integrand such that $\operatorname{dom} I_{\varphi}=L^{\infty}(\Omega, X)$ and there exists $v^{*} \in L^{1}\left(\Omega, X_{w^{*}}^{*}\right)$ such that $\int_{\Omega} \varphi^{*}\left(\omega, v^{*}(\omega)\right) d \mu(\omega)<\infty$, then $\Phi^{*}\left(x^{*}\right)=\int_{\Omega} \varphi^{*}\left(\omega, \hat{v}^{*}(\omega)\right) d \mu(\omega)$ for some $\hat{v}^{*} \in$ $L^{1}\left(\Omega, X_{w^{*}}^{*}\right)$ such that $x^{*}=\int_{\Omega} \hat{v}^{*}(\omega) d \mu(\omega)$ (here the integral of $\hat{v}^{*}(\cdot)$ is the Gelfand integral; see Diestel-Uhl [7], p. 53).

Proof. Let $A: X \rightarrow L^{\infty}(\Omega, X)$ be defined by $(A x)(\cdot)=x$ and $B: L^{1}\left(\Omega, X_{w^{*}}^{*}\right) \rightarrow$ $X^{*}$ by $B v^{*}=\int_{\Omega} v^{*}(\omega) d \mu(\omega)$ (the integral of $v^{*}(\cdot)$ being the Gelfand integral). Then if by $((\cdot, \cdot))$ we denote the duality brackets for the pair $\left(L^{\infty}(\Omega, X), L^{1}\left(\Omega, X_{w^{*}}^{*}\right)\right)$ (i.e. $\left(\left(y, v^{*}\right)\right)=\int_{\Omega}\left(y(\omega), v^{*}(\omega)\right) d \mu(\omega)$ for all $\left.\left[y, v^{*}\right] \in L^{\infty}(\Omega, X) \times L^{1}\left(\Omega, X_{w^{*}}^{*}\right)\right)$, we have

$$
\begin{aligned}
\left(\left(A x, v^{*}\right)\right) & =\int_{\Omega}\left(x, v^{*}(\omega)\right) d \mu(\omega)=\left(x, \int_{\Omega} v^{*}(\omega) d \mu(\omega)\right)=\left(x, B v^{*}\right) \\
\Rightarrow B & =\left.A^{*}\right|_{L^{1}\left(\Omega, X_{w^{*}}^{*}\right)}
\end{aligned}
$$

Now note that

$$
\Phi(x)=I_{\varphi} A(x) \Rightarrow \Phi^{*}\left(x^{*}\right)=\left(I_{\varphi} A\right)^{*}\left(x^{*}\right) .
$$

Clearly $A$ is continuous into $L^{\infty}(\Omega, X)$ furnished with the Mackey topology $m\left(L^{\infty}(\Omega, X), L^{1}\left(\Omega, X_{w^{*}}^{*}\right)\right)$. Also recalling that $X_{w^{*}}^{*}$ is a Souslin space, from Theorem VII-7 of Castaing-Valadier [6], we know that for the dual pair $\left(L^{1}\left(\Omega, X_{w^{*}}^{*}\right)\right.$, $\left.L^{\infty}(\Omega, X)\right)$ we have $\left(I_{\varphi^{*}}\right)^{*}=I_{\varphi^{* *}}=I_{\varphi} \Rightarrow I_{\varphi}$ is $m\left(L^{\infty}(\Omega, X), L^{1}\left(\Omega, X_{w^{*}}^{*}\right)\right)$-l.s.c. and $\operatorname{dom} I_{\varphi}=L^{\infty}(\Omega, X)$. Thus from Corollary 6.28, p. 335 of Laurent [16], we know that $I_{\varphi}(\cdot)$ is $m\left(L^{\infty}(\Omega, X), L^{1}\left(\Omega, X_{w^{*}}^{*}\right)\right)$-continuous. So we can apply Theorem $3, \mathrm{p}$. 179 of Ioffe-Tichomirov [13] and get that

$$
\begin{aligned}
\Phi^{*}\left(x^{*}\right) & =\left(I_{\varphi} A\right)^{*}\left(x^{*}\right)=\left(B I_{\varphi^{*}}\right)\left(x^{*}\right) \\
= & \inf \left\{I_{\varphi^{*}}\left(v^{*}\right): v^{*} \in L^{1}\left(\Omega, X_{w^{*}}^{*}\right), x^{*}=\int_{\Omega} v^{*}(\omega) d \mu(\omega)\right\}
\end{aligned}
$$

and furthermore there exists $\hat{v}^{*} \in L^{1}\left(\Omega, X_{w^{*}}^{*}\right)$ at which the above minimum is realized. Therefore

$$
\Phi^{*}\left(x^{*}\right)=\int_{\Omega} \varphi^{*}\left(\omega, \hat{v}^{*}(\omega)\right) d \mu(\omega), x^{*}=\int_{\Omega} \hat{v}^{*}(\omega) d \mu(\omega) .
$$


Remark. So the above proposition tells us that under its hypotheses, $\Phi^{*}(\cdot)=$ $\left(\oint_{\Omega} \varphi^{*}(\omega) d \mu(\omega)\right)(\cdot)$ and the infimum involved in the definition of the continuous infimal convolution (see section 2 ) is realized.

We will use the above conjugation result to prove a formula for the $\varepsilon$ subdifferential of $\Phi(\cdot)$.

Theorem 3. If $\varphi: \Omega \times X \rightarrow \overline{\mathbb{R}}=\mathbb{R} \cup\{+\infty\}$ is a normal convex integrand with $\operatorname{dom} I_{\varphi}=L^{\infty}(\Omega, X)$, and there exists $v^{*}(\cdot) \in L^{1}\left(\Omega, X_{w^{*}}^{*}\right)$ such that

$$
\int_{\Omega} \varphi^{*}\left(\omega, v^{*}(\omega)\right) d \mu(\omega)<\infty
$$

then for any $\varepsilon \geq 0$ we have that

$$
\partial_{\varepsilon} \Phi(x)=\bigcup_{\varepsilon(\cdot) \in \mathcal{L}_{+}^{1}(\varepsilon)} \int_{\Omega} \partial_{\varepsilon(\omega)} \varphi(\omega, x) d \mu(\omega),
$$

where

$$
\mathcal{L}_{+}^{1}(\varepsilon)=\left\{\varepsilon(\cdot) \in L^{1}(\Omega): \varepsilon(\omega) \geq 0 \mu \text {-a.e., } \int_{\Omega} \varepsilon(\omega) d \mu(\omega)=\varepsilon\right\} .
$$

Proof. Directly from the definition of the $\varepsilon$-subdifferential (cf. section 2), we see that if $\varepsilon(\cdot) \in \mathcal{L}^{1}(\varepsilon)$, then $x^{*} \in \partial_{\varepsilon(\omega)} \varphi(\omega, x)$ if and only if $\varphi(\omega, x)+\varphi^{*}\left(\omega, x^{*}\right)-\varepsilon(\omega) \leq$ $\left(x^{*}, x\right)$. Set

$$
k\left(\omega, x^{*}\right)=\varphi^{*}\left(\omega, x^{*}\right)-\left(x^{*}, x\right) \quad \text { and } \quad r(\omega)=\varepsilon(\omega)-\varphi(\omega, x) .
$$

Hence we can write that $\partial_{\varepsilon(\omega)} \varphi(\omega, x)=\left\{x^{*} \in X^{*}: k\left(\omega, x^{*}\right) \leq r(\omega)\right\} \Rightarrow$ $\operatorname{Gr} \partial_{\varepsilon(\cdot)} \varphi(\cdot, x)=\left\{\left(\omega, x^{*}\right) \in \Omega \times X^{*}: k\left(\omega, x^{*}\right)-r(\omega) \leq 0\right\}$. Recalling that the convex conjugate of a normal integrand is also normal (for $X^{*}$ equipped with the $w^{*}$-topology), we get that $\left(\omega, x^{*}\right) \rightarrow k\left(\omega, x^{*}\right)$ is $\Sigma \times B\left(X_{w^{*}}^{*}\right)$-measurable. Hence $\operatorname{Gr} \partial_{\varepsilon(\cdot)} \varphi(\cdot, x) \in \Sigma \times B\left(X_{w^{*}}^{*}\right)$. Since $X_{w^{*}}^{*}$ is a Souslin space, we can apply the Aumann-Saint Beuve selection theorem (see Saint Beuve [28] or Wagner [30], Theorem 5.10), to get $x^{*}: \Omega \rightarrow X_{w^{*}}^{*}$ a measurable map such that $x^{*}(\omega) \in \partial_{\varepsilon(\omega)} \varphi(\omega, x)$ for all $\omega \in \Omega$. Then from the definition of the $\varepsilon(\omega)$-subdifferential, we have

$$
\left(x^{*}(\omega), y-x\right)-\varepsilon(\omega) \leq \varphi(\omega, y)-\varphi(\omega, x), \quad y \in X .
$$

First let $y=y^{\prime}+x, y^{\prime} \in X$. We have

$$
\left(x^{*}(\omega), y^{\prime}\right)-\varepsilon(\omega) \leq \varphi\left(\omega, y^{\prime}+x\right)-\varphi(\omega, x), \quad y^{\prime} \in X .
$$

Next let $y=x-y^{\prime}, y^{\prime} \in X$. Then

$$
\left(x^{*}(\omega),-y^{\prime}\right)-\varepsilon(\omega) \leq \varphi\left(\omega, x-y^{\prime}\right)-\varphi(\omega, x), \quad y^{\prime} \in X .
$$

It is then clear from (2) and (3) above that for all $y \in X, \omega \rightarrow\left(x^{*}(\omega), y\right) \in L^{1}(\Omega)$. Hence there is $x^{*} \in X^{*}$ such that $\left(x^{*}, y\right)=\int_{\Omega}\left(x^{*}(\omega), y\right) d \mu(\omega)$ for all $y \in X$; i.e. $x^{*}=\int_{\Omega} x^{*}(\omega) d \mu(\omega)$ (the Gelfand integral of $x^{*}(\cdot)$; see Diestel-Uhl [7], p. 53). Integrating inequality (1) above, we get

$$
\left(x^{*}, y-x\right)-\int_{\Omega} \varepsilon(\omega) d \mu(\omega) \leq \Phi(y)-\Phi(x) \quad \text { for all } y \in X .
$$

Recalling that $\varepsilon(\cdot) \in \mathcal{L}_{+}^{1}(\varepsilon)$, we conclude that $x^{*} \in \partial_{\varepsilon} \Phi(x)$. So we have that

$$
\bigcup_{\varepsilon(\cdot) \in \mathcal{L}_{+}^{1}(\varepsilon)} \int_{\Omega} \partial_{\varepsilon(\omega)} \varphi(\omega, x) d \mu(\omega) \subseteq \partial_{\varepsilon} \Phi(x) .
$$


Next we will show that the opposite inclusion also holds. So let $x^{*} \in \partial \Phi_{\varepsilon}(x)$. Then we have

$$
\Phi(x)+\Phi^{*}\left(x^{*}\right)-\left(x^{*}, x\right) \leq \varepsilon .
$$

But from Proposition 2, we know that there exists $\hat{v}^{*} \in L^{1}\left(\Omega, X_{w^{*}}^{*}\right)$ with $x^{*}=$ $\int_{\Omega} \hat{v}^{*}(\omega) d \mu(\omega)$ and $\Phi^{*}\left(x^{*}\right)=\int_{\Omega} \varphi^{*}\left(\omega, \hat{v}^{*}(\omega)\right) d \mu(\omega)$. So we have

$$
\int_{\Omega} \varphi(\omega, x) d \mu(\omega)+\int_{\Omega} \varphi^{*}\left(\omega, \hat{v}^{*}(\omega)\right) d \mu(\omega)-\int_{\Omega}\left(\hat{v}^{*}(\omega), x\right) d \mu(\omega) \leq \varepsilon .
$$

Let $\varepsilon^{\prime}(\omega)=\varphi(\omega, x)+\varphi^{*}\left(\omega, \hat{v}^{*}(\omega)\right)-\left(\hat{v}^{*}(\omega), x\right)$. Obviously $\varepsilon^{\prime}(\cdot) \in L^{1}(\Omega)$ and $\varepsilon^{\prime}(\omega) \geq 0 \mu$-a.e. (Young-Fenchel inequality). Then $x^{*} \in \int_{\Omega} \partial_{\varepsilon^{\prime}(\omega)} \varphi(\omega, x) d \mu(\omega)$. Since clearly the $\varepsilon$-subdifferential increases with $\varepsilon$, we only need to consider $\varepsilon(\cdot) \in$ $\mathcal{L}_{+}^{1}(\varepsilon)$ and so

$$
\partial \Phi_{\varepsilon}\left(x^{*}\right) \subseteq \bigcup_{\varepsilon(\cdot) \in \mathcal{L}_{+}^{1}(\varepsilon)} \int_{\Omega} \partial_{\varepsilon(\omega)} \varphi(\omega, x) d \mu(\omega)
$$

From (4) and (5) we conclude that equality holds.

Remark. (1) If $\varepsilon=0$, then we recover Theorem 23(b) of Rockafellar [24], established there under the additional hypothesis that $X$ is reflexive.

(2) For $X=\mathbb{R}^{n}$, this formula for the $\varepsilon$-subdifferential of $\Phi(x)$ was first obtained by Hiriart-Urruty [9].

This leads us to a corresponding formula for the $\varepsilon$-directional derivative of $\Phi(\cdot)$ in terms of the $\varepsilon$-directional derivatives of the functions $\varphi(\omega, \cdot), \omega \in \Omega$.

Recall that if $\varphi \in \Gamma_{0}(X)$, then the $\varepsilon$-directional derivative of $\varphi(\cdot)$ is defined by

$$
\varphi_{\varepsilon}^{\prime}(x ; h)=\inf _{\lambda>0} \frac{\varphi(x+\lambda h)-\varphi(x)+\varepsilon}{\lambda} .
$$

Note that $\varphi_{\varepsilon}^{\prime}(x ; \cdot)$ is sublinear and $\varphi_{\varepsilon}^{\prime}(x ; \cdot)$ is the support function of $\partial_{\varepsilon} \varphi(x)$; i.e. $\sigma\left(h, \partial_{\varepsilon} \varphi(x)\right)=\sup \left[\left(x^{*}, h\right): x^{*} \in \partial_{\varepsilon} \varphi(x)\right]=\varphi_{\varepsilon}^{\prime}\left(x_{0} ; h\right)$ (see Rockafellar [26], pp. 219-220 or Hiriart-Urruty [9]).

Proposition 4. If $\varphi: \Omega \times X \rightarrow \overline{\mathbb{R}}=\mathbb{R} \cup\{+\infty\}$ is normal convex integrand, with $\operatorname{dom} I_{\varphi}=L^{\infty}(\Omega, X), I_{\varphi}(\cdot)$ is finite there and there exists $x^{*} \in L^{1}\left(\Omega, X_{w^{*}}^{*}\right)$ such that $\int_{\Omega} \varphi^{*}\left(\omega, x^{*}(\omega)\right) d \mu(\omega)<\infty$, then for all $\varepsilon \geq 0$ and all $(x, h) \in X \times X$ we have

$$
\Phi_{\varepsilon}^{\prime}(x ; h)=\sup \left[\int_{\Omega} \varphi_{\varepsilon(\omega)}^{\prime}(\omega, x ; h) d \mu(\omega): \varepsilon(\cdot) \in \mathcal{L}_{+}^{1}(\varepsilon)\right] .
$$

Proof. Using Theorem 3, we have

$$
\begin{aligned}
\Phi_{\varepsilon}^{\prime}(x ; h) & =\sigma\left(h, \partial_{\varepsilon} \Phi(x)\right) \\
& =\sup \left[\sigma\left(h, \int_{\Omega} \partial_{\varepsilon(\omega)} \varphi(\omega, x) d \mu(\omega)\right): \varepsilon(\cdot) \in \mathcal{L}_{+}^{1}(\varepsilon)\right]
\end{aligned}
$$


But note that from Theorem 2.2 of Hiai-Umegaki [8], we have that

$$
\begin{aligned}
\sigma(h & \left., \int_{\Omega} \partial_{\varepsilon(\omega)} \varphi(\omega, x) d \mu(\omega)\right)=\int_{\Omega} \sigma\left(h, \partial_{\varepsilon(\omega)} \varphi(\omega, x)\right) d \mu(\omega) \\
& =\int_{\Omega} \varphi_{\varepsilon(\omega)}^{\prime}(\omega, x ; h) d \mu(\omega) \\
& \Rightarrow \Phi_{\varepsilon}^{\prime}(x ; h)=\sup \left[\int_{\Omega} \varphi_{\varepsilon(\omega)}^{\prime}(\omega, x ; h) d \mu(\omega): \varepsilon(\cdot) \in \mathcal{L}_{+}^{1}(\varepsilon)\right]
\end{aligned}
$$

\section{Continuous infimal Convolution}

In this section we obtain a necessary and sufficient condition for an element $x(\cdot) \in$ $L^{1}(\Omega, X)$ to be an approximate solution to the continuous infimal convolution. As we already mentioned in section 2 , the operation of continuous infimal convolution arises in mathematical economics, in the analysis of problems involving transferable utilities. In fact, in this case $\varphi(\cdot, \cdot)$ being a utility function is concave in $x$, and the infimum in the operation is replaced by a supremum. Then the economic interpretation is the following: Think of $(\Omega, \Sigma, \mu)$ as the space of agents, with $\Sigma$ being the collection of all allowable coalitions and $\mu(\cdot)$ a measure, which measures the size of each coalition and which is nonatomic (this notion of a continuum space of agents was first introduced by Aumann [1], as a device that captures better the spirit of pure competition; the nonatomicity of $\mu(\cdot)$ reflects the fact that there are no dominant (oligopolistic) agents in the market). Also $X$ is the commodity space. Recently there has been a trend among mathematical economists to consider models with an infinite-dimensional commodity space. As with the case of the space of agents, an infinite-dimensional commodity space more properly depicts a situation where we deal with a finite yet large number of commodities. Then $\int_{A} \varphi(\omega, x(\omega)) d \mu(\omega)=I_{\varphi}^{A}(x)$ represents the aggregate utility of coalition $A \in \Sigma$ under the commodity assignment $x(\cdot)$. If $x \in X$ is the aggregate initial endowment given to coalition $A \in \Sigma$, then we want to find that commodity assignment which will maximize the aggregate utility; i.e. solve the problem

$$
\sup \left[\int_{\Omega} \varphi(\omega, v(\omega)) d \mu(\omega): v \in L^{1}(\Omega, X), x=\int_{\Omega} v(\omega) d \mu(\omega)\right] .
$$

Of course we can have $\Omega=T=[0, b], \Sigma=B(T)=$ the Borel $\sigma$-field of $T$ and $\mu=d t$ the Lebesgue measure. Then we think of $t$ as a time variable and $I_{\varphi}(v)$ is the intertemporal utility generated by commodity assignment $v(\cdot) \in L^{1}(T, X)$.

As before, let $(\Omega, \Sigma, \mu)$ be a $\sigma$-finite measure space and $X$ a separable Banach space.

Theorem 5. If $\varphi: \Omega \times X \rightarrow \overline{\mathbb{R}}=\mathbb{R} \cup\{+\infty\}$ is a normal convex integrand, $\hat{v}(\cdot) \in L^{1}(\Omega, X)$ with $\int_{\Omega} \hat{v}(\omega) d \mu(\omega)=x, \partial\left(\oint_{\Omega} \varphi(\omega) d \mu(\omega)\right)(x) \neq 0$ and $\varepsilon>0$, then $I_{\varphi}(\hat{v}) \leq\left(\oint_{\Omega} \varphi(\omega) d \mu(\omega)\right)(x)+\varepsilon$ if and only if there exist $x^{*} \in X^{*}$ and $\varepsilon(\cdot) \in L^{1}(\Omega)$, $\varepsilon(\omega) \geq 0 \mu$-a.e. such that $x^{*} \in \partial_{\varepsilon(\omega)} \varphi(\omega, \hat{v}(\omega))$ and $\int_{\Omega} \varepsilon(\omega) d \mu(\omega) \leq \varepsilon$.

Proof. Sufficiency: From the definition of the $\varepsilon$-subdifferential, we know that for any $y \in L^{1}(\Omega, X)$

$$
\begin{aligned}
& \left(x^{*}, y(\omega)-\hat{v}(\omega)\right)-\varepsilon(\omega) \leq \varphi(\omega, y(\omega))-\varphi(\omega, \hat{v}(\omega)) \mu \text {-a.e. } \\
& \quad \Rightarrow I_{\varphi}(\hat{v})+\left(x^{*}, \int_{\Omega}(y(\omega)-\hat{v}(\omega)) d \mu(\omega)\right)-\int_{\Omega} \varepsilon(\omega) d \mu(\omega) \leq I_{\varphi}(y) .
\end{aligned}
$$


Let $\mathcal{L}^{1}(x)$ be the subset of $L^{1}(\Omega, X)$ defined by

$$
\mathcal{L}^{1}(x)=\left\{y \in L^{1}(\Omega, X): x=\int_{\Omega} y(\omega) d \mu(\omega)\right\} .
$$

Taking the infimum of both sides of $(6)$ over $\mathcal{L}^{1}(x)$, we get

$$
\begin{aligned}
I_{\varphi}(\hat{v}) & -\int_{\Omega} \varepsilon(\omega) d \mu(\omega) \leq\left(\oint_{\Omega} \varphi(\omega) \mu(\omega)\right)(x) \\
& \Rightarrow I_{\varphi}(\hat{v})-\varepsilon \leq\left(\oint_{\Omega} \varphi(\omega) d \mu(\omega)\right)(x) \quad\left(\text { since } \int_{\Omega} \varepsilon(\omega) d \mu(\omega) \leq \varepsilon\right) .
\end{aligned}
$$

Necessity: Let $x^{*} \in \partial\left[\oint_{\Omega} \varphi(\omega) d \mu(\omega)\right](x)$ and consider the function

$$
u(\omega, y)=\varphi(\omega, \hat{v}(\omega))-\varphi(\omega, y)+\left(x^{*}, y-\hat{v}(\omega)\right) .
$$

Set $\varepsilon(\omega)=\sup [u(\omega, y): y \in X]$. Since $u(\cdot, \cdot)$ is clearly jointly measurable, $\varepsilon(\cdot)$ is measurable too (cf. Lemma 2.1 of Hiai-Umegaki [8]). Also by taking $y=\hat{v}(\omega)$, we see that $\varepsilon(\omega) \geq 0 \mu$-a.e. Then we have

$$
\begin{gathered}
\varphi(\omega, \hat{x}(\omega))-\varphi(\omega, y)+\left(x^{*}, y-\hat{v}(\omega)\right) \leq \varepsilon(\omega), \quad \omega \in \Omega, \\
\Rightarrow x^{*} \in \partial_{\varepsilon(\omega)} \varphi(\omega, \hat{v}(\omega)), \quad \omega \in \Omega .
\end{gathered}
$$
have

We claim that $\int_{\Omega} \varepsilon(\omega) d \mu(\omega) \leq \varepsilon$. Indeed since $x^{*} \in \partial\left(\oint_{\Omega} \varphi(\omega) d \mu(\omega)\right)(x)$, we

$$
\left(x^{*}, y-x\right) \leq\left(\oint_{\Omega} \varphi(\omega) d \mu(\omega)\right)(y)-(\oint \varphi(\omega) d \mu(\omega))(x) \quad y \in X .
$$

So for all $y(\cdot) \in L^{1}(\Omega, X)$, we have

$$
\begin{aligned}
& \left(x^{*}, \int_{\Omega}(y(\omega)-\hat{v}(\omega)) d \mu(\omega)\right) \leq I_{\varphi}(y)-I_{\varphi}(\hat{v})+\varepsilon \\
& \quad \Rightarrow \int_{\Omega}\left(\varphi(\omega, \hat{v}(\omega))-\varphi(\omega, y(\omega))+\left(x^{*}, y(\omega)-\hat{v}(\omega)\right)\right) d \mu(\omega) \leq \varepsilon \\
& \quad \Rightarrow \sup \left[\int_{\Omega}\left(\varphi(\omega, \hat{v}(\omega))-\varphi(\omega, y(\omega))+\left(x^{*}, y(\omega)-\hat{v}(\omega)\right)\right) d \mu(\omega):\right. \\
& \left.\quad y \in L^{1}(\Omega, X)\right] \leq \varepsilon \\
& \Rightarrow \int_{\Omega} \sup \left[\varphi(\omega, \hat{v}(\omega))-\varphi(\omega, y)+\left(x^{*}, y-\hat{v}(\omega)\right): y \in X\right] d \mu(\omega) \leq \varepsilon
\end{aligned}
$$

(cf. Rockafellar [25] or Hiai-Umegaki [8], Theorem 2.2). So finally we get

$$
\int_{\Omega} \varepsilon(\omega) d \mu(\omega) \leq \varepsilon
$$

Using Theorem 3, we can also have an expression for the level sets of the continuous infimal convolution functional $\left(\oint_{\Omega} \varphi(\omega) d \mu(\omega)\right)(\cdot)=G(\cdot)$.

Given a function $\varphi: X \rightarrow \mathbb{R} \cup\{ \pm \infty\}$ we define $L_{\lambda}^{\varphi}=\{x \in X: \varphi(x) \leq \lambda\}$, the $\lambda$-level set of $\varphi(\cdot)$.

Theorem 6. If $X^{*}$ is separable too, $\varphi: \Omega \times X \rightarrow \overline{\mathbb{R}}=\mathbb{R} \cup\{+\infty\}$ is a normal convex integrand with $\operatorname{dom} I_{\varphi^{*}}=L^{\infty}\left(\Omega, X^{*}\right), \varphi^{*}(\cdot, 0) \in L^{1}(\Omega)$ and there exists 
$v(\cdot) \in L^{1}(\Omega, X)$ such that $\int_{\Omega} \varphi(\omega, v(\omega)) d \mu(\omega)<\infty$. Then for all $\lambda \geq \inf [G(x)$ : $x \in X]$ we have

$$
L_{\lambda}^{G}=\bigcup_{\substack{\lambda(\cdot) \in \mathcal{L}_{+}^{1}(\lambda) \\ \inf _{x \in X} \varphi(\omega, x) \leq \lambda(\omega) \mu-a . e .}} \int_{\Omega} L_{\lambda(\omega)}^{\varphi(\omega, \cdot)} d \mu(\omega) .
$$

Proof. From Theorem 1.6 of Ioffe-Tihomirov [12], we know that

$$
G^{*}(0)=\left(\oint_{\Omega} \varphi(\omega) d \mu(\omega)\right)^{*}(0)=\int_{\Omega} \varphi^{*}(\omega, 0) d \mu(\omega)
$$

and is finite by hypothesis. Then

$$
\begin{aligned}
L_{\lambda}^{G} & =\{x \in X: G(x) \leq \lambda\}=\left\{x \in X: G(x)+G^{*}(0) \leq \lambda+G^{*}(0)\right\} \\
& =\partial_{\lambda+G^{*}(0)} G^{*}(0) .
\end{aligned}
$$

Since $X^{*}$ is separable, we can apply Theorem 3 and get that

$$
\partial_{\lambda+G^{*}(0)} G^{*}(0)=\bigcup_{\varepsilon(\cdot) \in \mathcal{L}_{+}^{1}\left(\lambda+G^{*}(0)\right)} \int_{\Omega} \partial_{\varepsilon(\omega)} \varphi^{*}(\omega, 0) d \mu(\omega) .
$$

As for $G^{*}(\cdot)$, we can also get that

$$
\partial_{\varepsilon(\omega)} \varphi^{*}(\omega, 0)=L_{\varepsilon(\omega)-\varphi^{*}(\omega, 0)}^{\varphi(\omega, \cdot} .
$$

So we have

$$
L_{\lambda}^{G}=\bigcup_{\varepsilon(\cdot) \in \mathcal{L}_{+}^{1}\left(\lambda+G^{*}(0)\right)} \int_{\Omega} L_{\varepsilon(\omega)}^{\varphi(\omega, \cdot)} d \mu(\omega) .
$$

Set $\lambda(\omega)=\varepsilon(\omega)-\varphi^{*}(\omega, 0)$. Then

$$
\begin{aligned}
\int_{\Omega} \lambda(\omega) d \mu(\omega) & =\int_{\Omega}\left(\varepsilon(\omega)-\varphi^{*}(\omega, 0)\right) d \mu(\omega) \\
& =\lambda+G^{*}(0)-\int_{\Omega} \varphi^{*}(\omega, 0) d \mu(\omega)=\lambda .
\end{aligned}
$$

Also recall that $-\varphi^{*}(\omega, 0)=\inf [\varphi(\omega, x): x \in X]$. So finally we have

$$
L_{\lambda}^{G}=\bigcup_{\substack{\lambda(\cdot) \in \mathcal{L}_{+}^{1}(\lambda) \\ \inf _{x \in X} \varphi(\omega, x) \leq \lambda(\omega) \mu \text {-a.e. }}} \int_{\Omega} L_{\lambda(\omega)}^{\varphi(\omega, \cdot)} d \mu(\omega) .
$$

Remark. Our result extends Proposition 32 of Hiriart-Urruty [9], where $X=\mathbb{R}^{n}$.

\section{COMmUtation OF CONDITIONAL EXPECTATION AND SUBDIFFERENTIATION}

Our aim in this section is to produce verifiable conditions which will guarantee that the operators of conditional expectation and subdifferentiation commute when applied to normal convex integrands. The first such result was proved by Rockafellar-Wets [27] for integrands defined on $\Omega \times \mathbb{R}^{n}$. Here we extend their result to integrands defined on $\Omega \times X$, with $X$ being a separable Banach space. Also our proof is different and is based on two auxiliary results which are actually of independent interest. 
So let $(\Omega, \Sigma, \mu)$ be a complete probability space, $\Sigma_{0} \subseteq \Sigma$ a sub- $\sigma$-field and $X$ a separable Banach space. Given a normal integrand $\varphi: \Omega \times X \rightarrow \overline{\mathbb{R}}=\mathbb{R} \cup\{+\infty\}$ satisfying $\omega \rightarrow \inf \{\varphi(\omega, x):\|x\| \leq n\}=\beta_{n}(\omega) \in L^{1}(\Omega)$ for every $n \geq 1$ (i.e. $\varphi(\cdot, \cdot)$ is quasi-integrable), there exists a $\Sigma_{0}$-normal integrand $\psi: \Omega \times X \rightarrow \overline{\mathbb{R}}=\mathbb{R} \cup\{+\infty\}$, such that

$$
\int_{A} \varphi(\omega, v(\omega)) d \mu(\omega)=\int_{A} \psi(\omega, v(\omega)) d \mu(\omega)
$$

for all $(A, v) \in \Sigma_{0} \times L^{\infty}\left(\Sigma_{0}, X\right)$. In addition $\psi(\cdot, \cdot)$ is unique up to sets of the form $N \times X, \mu(N)=0$. Then $\psi(\cdot, \cdot)$ is said to be the $\Sigma_{0}$-conditional expectation of $\varphi$ and we write $\psi=E^{\Sigma_{0}} \varphi$ (see Klei [14] and Thibault [29]).

The same can be said for integrands $\varphi^{*}: \Omega \times X^{*} \rightarrow \overline{\mathbb{R}}=\mathbb{R} \cup\{+\infty\}$ which are normal for a topology compatible with the duality $\left(X^{*}, X\right)$ and they satisfy the previous quasi-integrability hypothesis (cf. Klei [14], Theorem 3.6).

We start with a result concerning the conditional expectation of the directional derivative of a normal convex integrand.

Proposition 7. If $\varphi: \Omega \times X \rightarrow \overline{\mathbb{R}}=\mathbb{R}=\cup\{+\infty\}$ is a normal convex quasiintegrable integrand and $I_{\varphi}(\cdot)$ is norm continuous on $L^{\infty}(\Omega, X)$ at $x_{0}(\cdot) \in$ $L^{\infty}\left(\Sigma_{0}, X\right)$, then

$$
E^{\Sigma_{0}} \varphi^{\prime}\left(\omega, x_{0}(\omega) ; h\right)=\left(E^{\Sigma_{0}} \varphi\right)^{\prime}\left(\omega, x_{0}(\omega) ; h\right)
$$

for all $\omega \in \Omega \backslash N, \mu(N)=0$ and all $h \in X$.

Proof. Using the definition of the directional derivative we can readily check that $(\omega, h) \rightarrow \varphi^{\prime}\left(\omega, x_{0}(\omega) ; h\right)$ is a normal, convex (in fact, sublinear) integrand. Also we have that

$$
\varphi\left(\omega, x_{0}(\omega)\right)-\varphi\left(\omega, x_{0}(\omega)-h\right) \leq \varphi^{\prime}\left(\omega, x_{0}(\omega) ; h\right) \mu \text {-a.e. }
$$

Since by hypothesis $I_{\varphi}(\cdot)$ is norm continuous on $L^{\infty}(\Omega, X)$ at $x_{0}(\cdot)$, from Theorem 1, p. 24 of Ioffe-Levin [11], we know that there exists $\varepsilon>0$ such that for all $h \in X,\|h\| \leq \varepsilon$,

$$
\varphi\left(\omega, x_{0}(\omega)-h\right) \leq r(\omega)
$$

with $r(\cdot) \in L_{1}(\Omega)$. So

$$
\varphi\left(\omega, x_{0}(\omega)\right)-r(\omega) \leq \varphi^{\prime}\left(\omega, x_{0}(\omega) ; h\right) .
$$

Since $\varphi^{\prime}\left(\omega, x_{0}(\omega) ; \cdot\right)$ is sublinear, we deduce that for every $n \geq 1$

$$
\hat{r}_{n}(\omega) \leq \varphi^{\prime}\left(\omega, x_{0}(\omega) ; h\right)
$$

for all $h \in X,\|h\| \leq n$ and with $\hat{r}_{n}(\cdot) \in L^{1}(\Omega)$. So $(\omega, h) \rightarrow \varphi^{\prime}\left(\omega, x_{0}(\omega) ; h\right)$ is a normal sublinear and quasi-integrable integrand and thus $E^{\Sigma_{0}} \varphi^{\prime}\left(\omega, x_{0}(\omega) ; h\right)$ is well defined.

Similarly, since by hypothesis $\varphi(\omega, x)$ is a normal convex and quasi-integrable integrand, $E^{\Sigma_{0}} \varphi(\omega, x)$ exists is $\Sigma_{0}$-normal and also is convex in $x$ (cf. Proposition 15 of Thibault [29]). So $\left(E^{\Sigma_{0}} \varphi\right)^{\prime}\left(\omega, x_{0}(\omega) ; h\right)$ is a well-defined $\Sigma_{0}$-normal integrand. 
From the monotone convergence theorem, we have for any $(A, h) \in \Sigma_{0} \times$ $L^{\infty}\left(\Sigma_{0}, X\right)$

$$
\begin{aligned}
& \int_{A} \varphi^{\prime}\left(\omega, x_{0}(\omega) ; h(\omega)\right) d \mu(\omega) \\
&=\int_{A} \lim _{\lambda_{n} \downarrow 0} \frac{\varphi\left(\omega, x_{0}(\omega)+\lambda_{n} h(\omega)\right)-\varphi\left(\omega, x_{0}(\omega)\right)}{\lambda_{n}} d \mu(\omega) \\
&=\lim _{\lambda_{n} \downarrow 0} \int_{A} \frac{\varphi\left(\omega, x_{0}(\omega)+\lambda_{n} h(\omega)\right)-\varphi\left(\omega, x_{0}(\omega)\right)}{\lambda_{n}} d \mu(\omega) \\
&=\lim _{\lambda_{n} \downarrow 0} \int_{A} \frac{E^{\Sigma_{0}} \varphi\left(\omega, x_{0}(\omega)+\lambda_{n} h(\omega)\right)-E^{\Sigma_{0}} \varphi\left(\omega, x_{0}(\omega)\right)}{\lambda_{n}} d \mu(\omega) \\
&=\int_{A} \lim _{\lambda_{n} \downarrow 0} \frac{E^{\Sigma_{0}} \varphi\left(\omega, x_{0}(\omega)+\lambda_{n} h(\omega)\right)-E^{\Sigma_{0}} \varphi\left(\omega, x_{0}(\omega)\right)}{\lambda_{n}} d \mu(\omega) \\
&=\int_{A}\left(E^{\Sigma_{0}} \varphi\right)^{\prime}\left(\omega, x_{0}(\omega) ; h(\omega)\right) d \mu(\omega) \\
& \Rightarrow E^{\Sigma_{0}} \varphi^{\prime}\left(\omega, x_{0}(\omega) ; h\right)=\left(E^{\Sigma_{0}} \varphi\right)^{\prime}\left(\omega, x_{0}(\omega) ; h\right) \\
& \quad \text { for all } \omega \in \Omega \backslash N, \mu(N)=0 \text { and all } h \in X .
\end{aligned}
$$

The second auxiliary result that we will need is the following:

Proposition 8. If $X^{*}$ is separable too, $\varphi: \Omega \times X \rightarrow \overline{\mathbb{R}}=\mathbb{R} \cup\{+\infty\}$ is a normal, convex and quasi-integrable integrand and $I_{\varphi}(\cdot)$ is norm continuous on $L^{\infty}(\Omega, X)$ at $x_{0}(\cdot) \in L^{\infty}\left(\Sigma_{0}, X\right)$, then $E^{\Sigma_{0}} \partial \varphi\left(\omega, x_{0}(\omega)\right)=\partial E^{\Sigma_{0}} \varphi\left(\omega, x_{0}(\omega)\right) \mu$-a.e.

Proof. Since $I_{\varphi}(\cdot)$ is norm continuous at $x_{0}(\cdot)$, we have

$$
\left(I_{\varphi}\right)^{\prime}\left(x_{0} ; h\right)=\sigma\left(h, \partial^{*} I_{\varphi}\left(x_{0}\right)\right)
$$

for every $h \in L^{\infty}(\Omega, X)$ and with $\partial^{*} I_{\varphi}\left(x_{0}\right)$ denoting the subdifferential of $I_{\varphi}(\cdot)$ at $x_{0}$ in $L^{\infty}(\Omega, X)^{*}$.

As in the proof of Proposition 7, via the monotone convergence theorem, we can readily check that

$$
\left(I_{\varphi}\right)^{\prime}\left(x_{0} ; h\right)=\int_{\Omega} \varphi^{\prime}\left(\omega, x_{0}(\omega) ; h(\omega)\right) d \mu(\omega) \quad \text { for all } h \in L^{\infty}(\Omega, X) .
$$

Also from Theorem 1, p. 20 of Ioffe-Levin [11], we know that

$$
\partial^{*} I_{\varphi}\left(x_{0}\right)=S_{\partial \varphi\left(\cdot, x_{0}(\cdot)\right)}^{1}+N\left(x_{0} ; \operatorname{dom} I_{\varphi}\right)
$$

(here $N\left(x_{0} ; \operatorname{dom} I_{\varphi}\right)$ denotes the normal cone to $\operatorname{dom} I_{\varphi}$ at $\left.x_{0}\right)$. Since by hypothesis $I_{\varphi}(\cdot)$ is norm continuous on $L^{\infty}(\Omega, X)$ at $x_{0}$, we have $x_{0} \in \operatorname{int} \operatorname{dom} I_{\varphi}$ and so $N\left(x_{0} ; \operatorname{dom} I_{\varphi}\right)=\{0\}$. Therefore

$$
\begin{gathered}
\partial^{*} I_{\varphi}\left(x_{0}\right)=S_{\partial \varphi\left(\cdot, x_{0}(\cdot)\right)}^{1} \neq \varnothing \quad\left(\text { since } I_{\varphi}(\cdot) \text { is norm continuous at } x_{0}\right) \\
\Rightarrow \sigma\left(h, \partial^{*} I_{\varphi}\left(x_{0}\right)\right)=\sup \left[\left(\left(h, x^{*}\right)\right): x^{*} \in S_{\partial \varphi\left(\cdot, x_{0}(\cdot)\right)}^{1}\right] \\
=\int_{\Omega} \sup \left[\left(h(\omega), x^{*}\right): x^{*} \in \partial \varphi\left(\omega, x_{0}(\omega)\right)\right] d \mu(\omega) .
\end{gathered}
$$

This last equality follows from Theorem 2.2 of Hiai-Umegaki [8] and the observation that $\omega \rightarrow \partial \varphi\left(\omega, x_{0}(\omega)\right)$ is measurable since $\operatorname{Gr} \partial \varphi\left(\cdot, x_{0}(\cdot)\right)=\left\{\left(\omega, x^{*}\right) \in\right.$ $\left.\Omega \times X^{*}: \varphi\left(\omega, x_{0}(\omega)\right)+\varphi^{*}\left(\omega, x^{*}\right)=\left(x^{*}, x(\omega)\right)\right\} \in \Sigma \times B\left(X_{w^{*}}^{*}\right.$. So we have 


$$
\begin{aligned}
& \sigma\left(h, \partial^{*} I_{\varphi}\left(x_{0}\right)\right)=\int_{\Omega} \sigma\left(h(\omega), \partial \varphi\left(\omega, x_{0}(\omega)\right)\right) d \mu(\omega) \\
& \quad \Rightarrow \int_{A} \varphi^{\prime}\left(\omega, x_{0}(\omega) ; h(\omega)\right) d \mu(\omega)=\int_{A} \sigma\left(h(\omega), \partial \varphi\left(\omega, x_{0}(\omega)\right)\right) d \mu(\omega)
\end{aligned}
$$

for all $A \in \Sigma$ and all $h \in L^{\infty}(\Omega, X)$. Hence from Lemma 6 of Thibault [29], we get

$$
\begin{array}{r}
\varphi^{\prime}\left(\omega, x_{0}(\omega) ; h\right)=\sigma\left(h, \partial \varphi\left(\omega, x_{0}(\omega)\right)\right) \quad \text { for all } \omega \in \Omega \backslash N, \mu(N)=0 \text { and all } h \in X, \\
\Rightarrow E^{\Sigma_{0}} \varphi^{\prime}\left(\omega, x_{0}(\omega) ; h\right)=E^{\Sigma_{0}} \sigma\left(h, \partial \varphi\left(\omega, x_{0}(\omega)\right)\right) \\
\quad \text { for all } \omega \in \Omega \backslash N, \mu(N)=0 \text { and all } h \in X, \\
\Rightarrow\left(E^{\Sigma_{0}} \varphi\right)^{\prime}\left(\omega, x_{0}(\omega) ; h\right)=E^{\Sigma_{0}} \sigma\left(h, \partial \varphi\left(\omega, x_{0}(\omega)\right)\right) \\
\text { for all } \omega \in \Omega \backslash N, \mu(N)=0 \text { and all } h \in X,
\end{array}
$$

(cf. Proposition 7).

As before we can easily check that

$$
\begin{aligned}
& \left(E^{\Sigma_{0}} \varphi\right)^{\prime}\left(\omega, x_{0}(\omega) ; h\right)=\sigma\left(h, \partial E^{\Sigma_{0}} \varphi\left(\omega, x_{0}(\omega)\right)\right) \\
& \qquad \text { for all } \omega \in \Omega \backslash N, \mu(N)=0 \text { and all } h \in X .
\end{aligned}
$$

Then for every $(A, h) \in \Sigma_{0} \times L^{\infty}\left(\Sigma_{0}, X\right)$, we have

$$
\begin{aligned}
\int_{A} E^{\Sigma_{0}} \sigma\left(h(\omega), \partial \varphi\left(\omega, x_{0}(\omega)\right)\right) d \mu(\omega) \\
\quad=\int_{A} \sigma\left(h(\omega), \partial \varphi\left(\omega, x_{0}(\omega)\right)\right) d \mu(\omega) \\
\quad=\int_{A} \sup \left[\left(x^{*}, h(\omega)\right): x^{*} \in \partial \varphi\left(\omega, x_{0}(\omega)\right)\right] d \mu(\omega) \\
\quad=\sup \left[\int_{A}\left(v^{*}(\omega), h(\omega)\right) d \mu(\omega): v^{*} \in S_{\partial \varphi\left(\cdot, x_{0}(\cdot)\right)}^{1}=\partial^{*} I_{\varphi}\left(x_{0}\right)\right] \\
=\sup \left[\int_{A}\left(E^{\Sigma_{0}} v^{*}(\omega), h(\omega)\right) d \mu: v^{*} \in S_{\partial \varphi\left(\cdot, x_{0}(\cdot)\right)}^{1}\right] \\
=\int_{A} \sup \left[\left(y^{*}, h(\omega)\right): y^{*} \in E^{\Sigma_{0}} \partial \varphi\left(\omega, x_{0}(\omega)\right)\right] d \mu(\omega) \\
=\int_{A} \sigma\left(h(\omega) ; E^{\Sigma_{0}} \partial \varphi\left(\omega, x_{0}(\omega)\right)\right) d \mu(\omega) .
\end{aligned}
$$

Therefore we have that

$$
\begin{aligned}
& E^{\Sigma_{0}} \sigma\left(h ; \partial \varphi\left(\omega, x_{0}(\omega)\right)\right)=\sigma\left(h, E^{\Sigma_{0}} \partial \varphi\left(\omega, x_{0}(\omega)\right)\right) \\
& \quad \text { for all } \omega \in \Omega \backslash N, \mu(N)=0 \text { and all } h \in X, \\
& \Rightarrow \sigma\left(h, \partial E^{\Sigma_{0}} \varphi\left(\omega, x_{0}(\omega)\right)\right)=\sigma\left(h, E^{\Sigma_{0}} \partial \varphi\left(\omega, x_{0}(\omega)\right)\right) \\
& \quad \text { for all } \omega \in \Omega \backslash N, \mu(N)=0 \text { and all } h \in X .
\end{aligned}
$$

Since both sets are $w^{*}$-closed and convex, we conclude that

$$
\partial E^{\Sigma_{0}} \varphi\left(\omega, x_{0}(\omega)\right)=E^{\Sigma_{0}} \partial \varphi\left(\omega, x_{0}(\omega)\right) \mu \text {-a.e. }
$$

Now we are ready to state and prove our main theorem on the commutation of the conditional expectation and of the subdifferential operators.

Theorem 9. If $X^{*}$ is separable, $\varphi: \Omega \times X \rightarrow \overline{\mathbb{R}}=\mathbb{R} \cup\{+\infty\}$ is a normal convex and quasi-integrable integrand such that $\operatorname{dom} I_{\varphi}=L^{\infty}(\Omega, X)$ and $I_{\varphi}$ is norm contin- 
uous on $L^{\infty}(\Omega, X)$ at $x_{0}(\cdot) \in L^{\infty}\left(\Sigma_{0}, X\right)$, then $E^{\Sigma_{0}} \partial \varphi(\omega, x(\omega))=\partial E^{\Sigma_{0}} \varphi(\omega, x(\omega))$ $\mu$-a.e. for all $x(\cdot) \in L^{\infty}\left(\Sigma_{0}, X\right)$.

Proof. Since $I_{\varphi}(\cdot)$ is norm continuous on $L^{\infty}(\Omega, X)$ at $x_{0}(\cdot)$, it is bounded above in a strong neighborhood of $x_{0}(\cdot)$. So Theorem 6.2.7, p. 333 of Laurent [16] tells us that $I_{\varphi}(\cdot)$ is norm continuous on $\operatorname{int} \operatorname{dom} I_{\varphi}=L^{\infty}(\Omega, X)$. Hence we can apply Proposition 8 and get the desired conclusion.

\section{Conditional expectation of RAndom SETS}

A normal integral of particular interest is the indicator function of a random set. Specifically, let $F: \Omega \rightarrow P_{f}(X)$ be a measurable multifunction and define $\delta_{F(\omega)}(x)=0$ if $x \in F(\omega)$ and $\delta_{F(\omega)}(x)=+\infty$ if $x \notin F(\omega)$. Then clearly $(\omega, x) \rightarrow \delta_{F(\omega)}(x)$ is a normal integrand and $I_{\delta_{F}}=\delta_{S_{F}^{1}}$. Furthermore, if $F(\cdot)$ is $P_{f c}(X)$-valued, then $\delta_{F(\omega)}(x)$ is a normal convex integrand. Recalling that the convex conjugate of a normal integrand is a normal integrand and since $\left(\delta_{F(\omega)}\right)^{*}(\cdot)=$ $\sigma(\cdot, F(\omega))$ (the support function of $F(\omega)$ ), we have that $\left(\omega, x^{*}\right) \rightarrow \sigma\left(x^{*}, F(\omega)\right)$ is normal integrand and $I_{\sigma(\cdot, F)}=\sigma\left(\cdot, S_{F}^{1}\right)$. These two integrands are useful in stochastic optimization problems (see Rockafellar [25] and Rockafellar-Wets [27]) and in the study of the convergence properties of discrete-time, set-valued random processes (see Papageorgiou $[19,20]$ ). In this section we relate the conditional expectation of those integrands with the conditional expectation of the multifunction (random set) $F(\cdot)$.

Here $(\Omega, \Sigma, \mu)$ is complete, probability space, $\Sigma_{0}$ a sub $\sigma$-field of $\Sigma$ and $X$ a separable Banach space.

Proposition 10. If $F: \Omega \rightarrow P_{f c}(X)$ is a measurable multifunction with $S_{F}^{1} \neq \varnothing$, then $E^{\Sigma_{0}} \sigma\left(x^{*}, F(\omega)\right)=\sigma\left(x^{*}, E^{\Sigma_{0}} F(\omega)\right)$ for all $\omega \in \Omega \backslash N, \mu(N)=0$ and all $x^{*} \in$ $X^{*}$.

Proof. Let $x(\cdot) \in L^{\infty}\left(\Sigma_{0}, X_{w^{*}}^{*}\right)$ and $A \in \Sigma_{0}$. Then from Klei [14] we know that

$$
\begin{aligned}
\int_{A} E^{\Sigma_{0}} \sigma\left(x^{*}(\omega), F(\omega)\right) d \mu(\omega)=\int_{A} \sigma\left(x^{*}, F(\omega)\right) d \mu(\omega) \\
=\int_{A} \sup \left[\left(x^{*}(\omega), x\right): x \in F(\omega)\right] d \mu(\omega) \\
=\sup \left[\int_{A}\left(x^{*}(\omega), f(\omega)\right) d \mu(\omega): f \in S_{F}^{1}\right]
\end{aligned}
$$

(see Theorem 2.2 of Hiai-Umegaki [8])

$$
\begin{aligned}
& =\sup \left[\int_{A}\left(x^{*}(\omega), E^{\Sigma_{0}} f(\omega)\right) d \mu(\omega): f \in S_{F}^{1}\right] \\
& =\sup \left[\int_{A}\left(x^{*}(\omega), g(\omega)\right) d \mu(\omega): g \in S_{E^{\Sigma_{0} F}}^{1}\right] \\
& \qquad \quad\left(\text { since } S_{E^{\Sigma_{0} F}}^{1}={\overline{E^{\Sigma_{0}} S_{F}^{1}}}^{L^{1}\left(\Sigma_{0}, X\right)} ;\right. \text { see section 2) } \\
& =\int_{A} \sup \left[\left(x^{*}(\omega), y\right): y \in E^{\Sigma_{0}} F(\omega)\right] d \mu(\omega) \\
& =\int_{A} \sigma\left(x^{*}(\omega), E^{\Sigma_{0}} F(\omega)\right) d \mu(\omega) .
\end{aligned}
$$


From the uniqueness of the conditional expectation of normal integrands on $\Omega \times X^{*}$ up to sets $N \times X^{*}, \mu(N)=0$ (see Klei [14] and section 5), we get

$$
\begin{aligned}
& E^{\Sigma_{0}} \sigma\left(x^{*}, F(\omega)\right)=\sigma\left(x^{*}, E^{\Sigma_{0}} F(\omega)\right) \\
& \text { for all } \omega \in \Omega \backslash N, \mu(N)=0 \text { and all } x^{*} \in X^{*} .
\end{aligned}
$$

A similar result also holds for the indicator function.

Proposition 11. If $F: \Omega \rightarrow P_{f}(X)$ is a measurable multifunction with $S_{F}^{1} \neq \varnothing$, then $E^{\Sigma_{0}} \delta_{F(\omega)}(x)=\delta_{E^{\Sigma_{0} F(\omega)}}(x)$ for all $\omega \in \Omega \backslash N, \mu(N)=0$ and all $x \in X$.

Proof. Note that if $x(\cdot) \in S_{F}^{1}$, then $\delta_{F(\omega)}(x(\omega))=0 \mu$-a.e. So we can apply Proposition 6.2 of Levin [18] (see also Theorem 2 of Rockafellar [23]) and get that for all $A \in \Sigma_{0}$ and all $x^{*}(\cdot) \in L^{\infty}\left(\Sigma_{0}, X_{w^{*}}^{*}\right)$, we have

$$
\begin{aligned}
\left(I_{\delta_{F}}^{A}\right)^{*}\left(x^{*}\right) & =\int_{A} \sigma\left(x^{*}(\omega), F(\omega)\right) d \mu(\omega)=\int_{A} E^{\Sigma_{0}} \sigma\left(x^{*}(\omega), F(\omega)\right) d \mu(\omega) \\
& =\int_{A} \sigma\left(x^{*}, E^{\Sigma_{0}} F(\omega)\right) d \mu(\omega) \text { (cf. Proposition 10). }
\end{aligned}
$$

Since by definition $I_{\delta_{F}}^{A}=I_{E^{\Sigma_{0} \delta_{F}}}^{A}$, we have

$$
\left(I_{E^{\Sigma_{0} \delta_{F}}}^{A}\right)^{*}\left(x^{*}\right)=\int_{A} \sigma\left(x^{*}, E^{\Sigma_{0}} F(\omega)\right) d \mu(\omega)=I_{\sigma\left(\cdot, E^{\left.\Sigma_{0} F\right)}\right.}^{A}\left(x^{*}\right) .
$$

By taking conjugates within the dual pair $\left(L^{1}(\Omega, X), L^{\infty}\left(\Omega, X_{w^{*}}^{*}\right)\right)$, we get for all $x \in L^{1}\left(\Sigma_{0}, X\right)$

$$
\begin{aligned}
& \left(I_{E^{\Sigma_{0} \delta_{F}}}^{A}\right)(x)=\left(I_{\delta_{E^{\Sigma_{0}}}}^{A}\right)(x) \\
& \Rightarrow \int_{A} E^{\Sigma_{0}} \delta_{F(\omega)}(x(\omega)) d \mu(\omega)=\int_{A} \delta_{E^{\Sigma_{0} F(\omega)}}(x(\omega)) d \mu(\omega) \\
& \Rightarrow E^{\Sigma_{0}} \delta_{F(\omega)}(x)=\delta_{E^{\Sigma_{0} F(\omega)}}(x) \\
& \quad \text { for all } \omega \in \Omega \backslash N, \mu(N)=0 \text { and all } x \in X .
\end{aligned}
$$

\section{ACKNOWLEDGEMENT}

The author wishes to thank the anonymous referee for his (her) comments and criticisms.

\section{REFERENCES}

1. R. Aumann, Markets with a continuum of traders, Econometrica 32 (1964), 39-50. MR 30:2908

2. $\ldots$ Integrals of set-valued functions, J. Math. Anal. Appl. 12 (1965), 1-12. MR 32:2543

3. R. Aumann and L. Shapley, Values of Nonatomic Games, Princeton Univ. Press, Princeton, 1974.

4. J.-M. Bismut, Integrales convexes et probabilités, J. Math. Anal. Appl. 42 (1973), 639-673. MR 48:3083

5. N. Bourbaki, Espaces Vectoriels Topologiques, Hermann, Paris, 1966. MR 34:3277

6. C. Castaing and M. Valadier, Convex Analysis and Measurable Multifunctions, Springer, Berlin, 1977. MR 57:7169

7. J. Diestel and J. Uhl, Vector Measures, Math Surveys, Vol. 15, AMS, Providence, RI, 1977. MR 56:12216

8. F. Hiai and H. Umegaki, Integrals, conditional expectations and martingales of multivalued functions, J. Multiv. Anal. 7 (1977), 149-182. MR 58:22463

9. J.-B. Hiriart-Urruty, Thèse d'Etat, Chapter IV, Clermond-Ferrand, France, 1977. 
10. , Lipschitz $r$-continuity of the approximate subdifferential of a convex function, Math Scand. 47 (1980), 123-134. MR 82c:58007

11. A. Ioffe and V. Levin, Subdifferentials and convex functions, Trans. Moscow Math. Soc. 26 (1972), 1-72. MR 51:8817

12. A. Ioffe and V. Tihomirov, The duality of convex functions and extremal problems, Russian Math. Surveys 23 (1968), 53-124. MR 44:5797

13.

14. H.-A. Klei, Thèse d'Etat, Université de Paris VI, Paris, 1985.

15. N. Komuro, Basic properties of convex functions and convex integrands, Hokkaido Math. Jour. 18 (1989), 1-30. MR 90f:49008

16. P.-J. Laurent, Approximation et Optimisation, Hermann, Paris, 1972. MR 57:6947

17. V. Levin, The Lebesgue decomposition for functionals on the vector-function space $L_{X}^{\infty}$, Functional Anal. Appl. 8 (1974), 314-317. MR 57:850

18. Convex integral functionals and the theory of lifting, Russian Math. Surveys 30 (1975), 115-178. MR 53:3698

19. N. S. Papageorgiou, Convergence and representation theorems for set-valued random processes, J. Math. Anal. Appl. 150 (1990), 129-145. MR 92a:60117

20. W Weak convergence of random sets in Banach spaces, J. Math. Anal. Appl. 164 (1992), 571-589. MR 92m:49013

21. R. T. Rockafellar, Integrals which are convex functionals I, Pacific J. Math. 24 (1968), 525539. MR 38:4984

22. _ Integrals which are convex functionals II, Pacific J. Math. 39 (1971), 439-469. MR 46:9710

23. Analysis, ed. by E. Zarantonello, Academic Press, New York, 1971, pp. 215-236. MR 52:11693

24. Conjugate Duality and Optimization, Reg. Conf. Series in Appl. Math., vol. 16, SIAM, Philadelphia, 1973. MR 51:9811

25. Integral functionals, normal integrands and measurable multifunctions, in Nonlinear Operators and the Calculus of Variations, ed. by J.-P. Gossez et al., Springer, New York, 1976, pp. 157-207. MR 58:23598

26. Convex Analysis, Princeton Univ. Press, Princeton, 1970. MR 43:445

27. R. T. Rockafellar and R. J.-B. Wets, On the interchange of subdifferentiation and conditional expectation for convex functionals, Stochastics 10 (1982), 173-182. MR 83j:49022

28. M.-F. Saint-Beuve, On the extension of von Neumann-Aumann theorem, J. Funct. Anal. 17 (1974), 112-129. MR 51:10564

29. L. Thibault, Esperance conditionelle d'integrandes semicontinus, Ann. Inst. H. Poincare 17 (1981), 337-350. MR 83a:60005

30. M. Valadier, Integration de convexes fermes notamment d'epigraphes et inf-convolution continue, RAIRO 4 (1970), 57-73. MR 43:3881

31. D. Wagner, Survey of measurable selection theorems, SIAM J. Control. Optim. 15 (1977), 859-903. MR 58:6137

Department of Mathematics, National Technical University, Zografou Campus, Athens 15780, Greece 